\title{
Harmonics Generation by Surface Plasmon Polaritons on Single Nanowires
}

de Hoogh, Anouk; Opheij, Aron; Wulf, Matthias; Rotenberg, Nir; Kuipers, L.

\section{Published in:}

ACS Photonics

DOI:

10.1021/acsphotonics.5b00686

Publication date:

2016

Document version

Publisher's PDF, also known as Version of record

Document license:

CC BY-NC

Citation for published version (APA):

de Hoogh, A., Opheij, A., Wulf, M., Rotenberg, N., \& Kuipers, L. (2016). Harmonics Generation by Surface Plasmon Polaritons on Single Nanowires. ACS Photonics, 3(8), 1446-1452.

https://doi.org/10.1021/acsphotonics.5b00686 


\title{
Harmonics Generation by Surface Plasmon Polaritons on Single Nanowires
}

\author{
Anouk de Hoogh, Aron Opheij, Matthias Wulf, Nir Rotenberg, and L. Kuipers* \\ FOM Institute AMOLF, Science Park 104, 1098 XG, Amsterdam, The Netherlands \\ Supporting Information
}

ABSTRACT: We present experimental observations of visible wavelength second- and third-harmonic generation on single plasmonic nanowires of variable widths. We identify that nearinfrared surface plasmon polaritons, which are guided along the nanowire, act as the source of the harmonics generation. We discuss the underlying mechanism of this nonlinear process, using a combination of spatially resolved measurements and numerical simulations to show that the visible harmonics are generated via a combination of both local and propagating plasmonic modes. Our results provide the first demonstration of nanoscale nonlinear optics with guided, propagating plasmonic modes on a lithographically defined chip, opening up new routes toward integrated optical circuits for information processing.

KEYWORDS: nonlinear plasmonics, nanowires, harmonics generation

$\mathrm{N}$ onlinear optics is of tremendous importance to modern, light-based technology. ${ }^{1}$ Nonlinear optical phenomena, which lead to all-optical control of light fields or generation of new colors of light, are at the heart of applications ranging from laser-based techniques ${ }^{2}$ and microscopy ${ }^{3}$ to optical and quantum information processing. ${ }^{4}$ The recent use of nanophotonics to bring nonlinear optics to the nanoscale ${ }^{5,6}$ has allowed researchers to migrate these technologies to compact, integrated optical chips. Moreover, as these devices have shrunk, the concentration of the light fields has necessarily grown, enhancing the electric fields and boosting inherently weak nonlinear effects. ${ }^{7,8}$

Plasmonic platforms, known for confining fields to highly subwavelength dimensions, are promising candidates for bringing nonlinear optics to the nanoscale. ${ }^{9,10}$ Research in this area has, to date, mainly focused on the use of localized surface plasmon resonances, which are found on metallic nanostructures, to enhance nonlinear effects. ${ }^{11-16}$ For example, using plasmonic nanoantennas researchers have demonstrated efficient harmonic generation, sum-frequency generation, and four-wave mixing. ${ }^{13,17}$ Likewise, coupled gold nanoparticles have been used to greatly increase the efficiencies of four-wave mixing. ${ }^{12}$ Moreover, harmonic generation has been observed during transmission through a metallic hole array, where the aspect ratio of the hole controls the generation efficiency. ${ }^{18}$ In contrast, little work has been done on nonlinear optics with surface plasmon polaritons (SPPs), plasmonic modes that propagate. What has been demonstrated has been mainly limited to interface SPPs, ${ }^{19,20}$ which are confined in only one dimension, or to the tip of a taper. ${ }^{21,22}$ A study of nonlinear effects using plasmonic modes of waveguides such as nanowires (NWs), with a well-defined, uniform guided mode that is confined to a small cross-sectional area, is, to date, lacking. Nonlinear processes on such structures could be very beneficial, as they can support extremely high bandwidth pulses, ${ }^{10,23}$ allowing for high-intensity operation at low powers.

Here, we present the first experimental observations of a nonlinear optical effect-namely, harmonic generation-by guided SPPs on single $\mathrm{Au}$ NWs. We use a combination of spectral and spatial far-field microscopy to resolve both secondand third-harmonic generation (SHG and THG), using fundamental femtosecond pulses at $1550 \mathrm{~nm}$, on NWs with widths ranging from 90 to $550 \mathrm{~nm}$. We identify the guided fundamental SPPs as the source of the harmonic generation and discuss the processes that drive this nonlinear effect.

\section{METHOD}

To study whether guided SPPs can be used to generate harmonics, we use lithographically defined Au NWs on a BK7 substrate. We select this system, which is shown in Figure 1a, for several reasons. First, these NWs are known to support SPPs, and, importantly, the fundamental plasmonic mode has no cutoff; in fact, as the wire dimensions shrink, the mode is increasingly confined to the metal, becoming more plasmonic in nature (see Figure $1 \mathrm{~b}$ ). ${ }^{24}$ Second, we can efficiently couple free-space radiation to the NW SPPs, first using a grating to excite glass-Au interface SPPs, which are subsequently adiabatically transformed to NW SPPs with a taper. ${ }^{25,26}$ Finally, these $\mathrm{Au}$ NWs can be fabricated in a highly controlled and

Special Issue: Nonlinear and Ultrafast Nanophotonics

Received: December 1, 2015

Published: June 8, 2016 


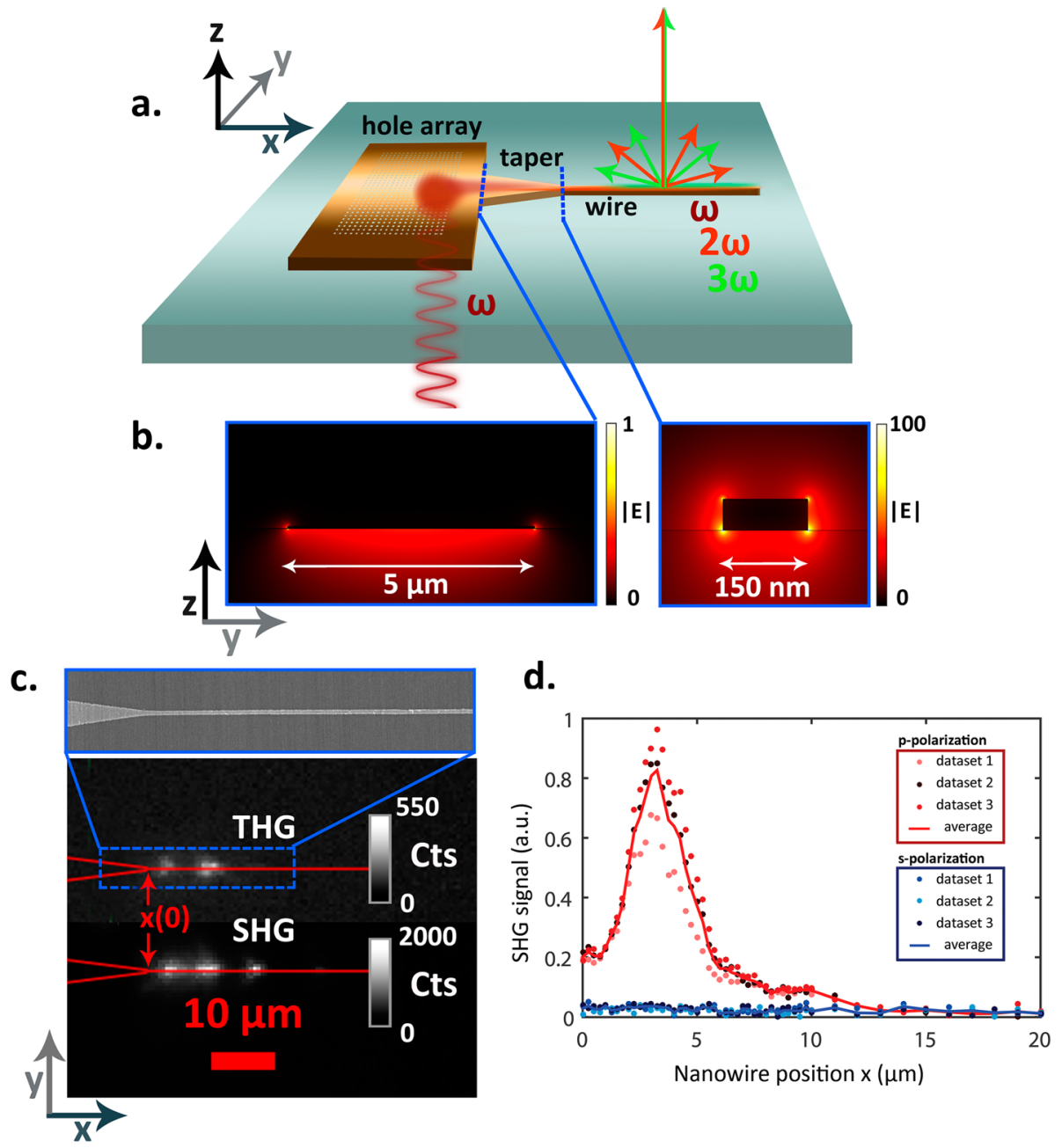

Figure 1. Harmonics generation on single Au nanowires on a glass substrate. (a) Schematic representation of the experiment. A hole array is illuminated from the $\mathrm{Au}$-glass interface with p-polarized femtosecond pulsed laser light, with a central wavelength $\lambda=1550 \mathrm{~nm}$. The interface SPPs, which propagate in the $x$ direction, are adiabatically converted to NW SPPs by gradually reducing the width of the wire. ${ }^{26}$ Visible light that scatters from the wire is collected on the Au-air side. (b) Normalized electric field distributions obtained from FEM simulations of the fundamental SPP mode for a cross section of the nanowire with a thickness $t=50 \mathrm{~nm}$ and a width $w=5 \mu \mathrm{m}$ (left) and $w=150 \mathrm{~nm}$ (right). (c) Examples of scattered visible light maps at 480-520 nm (top) and 750-800 nm (bottom), measured with a CCD camera. The scale bars represent spectrometer counts (Cts) per $5 \mathrm{~min}$. The red overlay shows the end of the taper and the wire to guide the eye. Top blue box: typical electron micrograph of a Au NW ( $w$ $=150 \mathrm{~nm}$ ). (d) SHG signal as a function of position for both p- and s-polarized excitations, shown with red and blue symbols, respectively. Although the excitation power of the s-polarized light is 3.75 times larger than that of the p-polarized light, SHG is observed only for the latter.

reproducible manner using electron-beam lithography, enabling the development of nanoscale optical circuitry. Despite the centrosymmetric character of $\mathrm{Au}$, it is reasonable to also expect second-order nonlinearities (e.g., SHG) when the structural symmetry is broken. ${ }^{27-29}$ Such symmetry breaking occurs at each surface of the NW where, conveniently, the intensities of the plasmonic mode are highest. At the same time, the symmetry of the NW geometry, as a whole, is also broken by the presence of the substrate. For our experiments, to study the effect of wire geometry on the harmonic generation, we fabricate $50 \mathrm{~nm}$ thick wires with widths ranging from 90 to 550 nm.

To achieve the high intensities required for nonlinear optical experiments, we use an $80 \mathrm{MHz}$ repetition rate, $120 \mathrm{fs}$ pulsed Ti:sapphire laser. This laser light is passed through an optical parametric oscillator, resulting in pulses with a central frequency of $1550 \mathrm{~nm}$, which we use to illuminate the grating (Figure 1a). As explained above, the resultant interface SPPs are then adiabatically converted to guided NW SPPs. A small fraction of the guided light on the NWs (either the fundamental or any harmonics) will be scattered off the wire by residual surface roughness or fabrication imperfections. We collect the light that is scattered perpendicularly to the sample surface with a 0.65 NA microscope objective and send it either to a spectrometer or to a CCD camera. In the latter case, we use a combination of filters to first spectrally select the frequency ranges of the harmonics (480-520 nm and 750-800 nm for the third- and second-harmonics generation, respectively). The resulting CCD images have a spatial resolution of $0.54 \mu \mathrm{m}$ per pixel. We note that in our setup the fundamental cannot be mapped in this way, as, near the beginning of the NW, the residual signal from the incident laser beam is much brighter than the scattered fundamental SPPs; see Supporting Information section 1. In the Supporting Information, we also discuss several alternative strategies, which could potentially be used as a reference to the harmonic intensities. When using the spectrometer, a slit is used to ensure that we collect light that originates only from the NW. 
In addition to the experiments, we also perform finiteelement simulations (using the mode solver in COMSOL multiphysics) to study the modes of the $\mathrm{Au}$ NWs. In these calculations, we use a refractive index of the BK7 glass substrate of 1.5 and take the complex refractive index of $\mathrm{Au}$ from literature. ${ }^{30}$ From these calculations we can extract both the properties of the plasmonic modes (e.g., their effective mode index or propagation length) and their mode profiles. Examples of mode profiles, for the beginning and end of the taper, are presented in Figure $1 \mathrm{~b}$. Here, we observe that the gradual decrease of the NW width from $5 \mu \mathrm{m}$ down to $150 \mathrm{~nm}$ results in electromagnetic field enhancements at the metal surface by a factor of $\sim 15$.

\section{RESULTS}

Having set out to detect harmonic generation from plasmonic NWs, we were pleasantly surprised to observe strong green light (as expected for the third harmonic of $1550 \mathrm{~nm}$ light) scattering from our sample while aligning our setup. By imaging the NW through a slit, we confirm that the source of the scattered, green light is indeed the NW and not, for example, the hole array.

When imaging our sample with different filters, we observe that the spatial maps of the scattered light (Figure 1c) for the green and red light, which are expected to correspond to thirdand second-harmonic generation respectively, have nearly identical spatial distributions in the intensity of THG and SHG along the nanowire. One noticeable difference, however, is that the third harmonic ( $\mathrm{TH})$ signal, whose generation is expected to scale with the third power of the fundamental intensity, fades more quickly along the NW than the second harmonic ( $\mathrm{SH}$ ) signal, which scales with as the second power. In the maps for a $150 \mathrm{~nm}$ wide NW, which we present in Figure 1c, we observe scattering for over $10 \mu \mathrm{m}$ along the NW. We observe $\mathrm{SH}$ and $\mathrm{TH}$ signal scattered along the wire, with distinguishable bright and dim areas. For a given nanowire, the locations of the bright and dim points of the $\mathrm{SH}$ and $\mathrm{TH}$ signal coincide, indicating that scattering of $\mathrm{SH}$ as well as $\mathrm{TH}$ seems to occur at the same points along the nanowire. However, the location of the bright points, and therefore the pattern of $\mathrm{SH}$ and $\mathrm{TH}$, differs from nanowire to nanowire. Even nominally identical wires exhibited seemingly random and distinct distributions of bright spots. This observation indicates that the scattering is due to random fabrication roughness. AFM measurements on four NWs with different widths reveal an average RMS value of 5-7 $\mathrm{nm}$. In scanning electron micrographs of these different NWs (an example of which is shown in the top panel of Figure 1c), we could not identify physical structures that would correlate with the observed optical patterns. Please note that as neither AFM nor SEM was able to identify clear scattering centers, they are either small, hard to identify in a trivial manner from geometry, or even located at the $\mathrm{Au}$-glass interface close enough to the sides of the nanowire that a signal in the collection direction could be generated. Furthermore, we envision that fabrication imperfections might even lie inside the NWs, due to the deposition process.

Next, we verify the plasmonic source of our visible signals by rotating the polarization of the incident light. We expect that visible light will be detected only when the sample is illuminated by p-polarized light (the only polarization that launches $\operatorname{SPPs}(\omega)$ on the NW) and not with s-polarized excitation, even when, in the latter case, significantly higher input powers are used. In Figure 1d, we show an example of the distributions of red light along the NW for the two polarizations. Indeed, as expected, this signal is visible only when SPPs are launched, confirming that the source of the visible light is plasmonic. Similar results (not shown) were obtained with the green light.

Finally, we observe that the distinct scattering pattern of a NW can be irreversibly altered by exceeding a certain illumination power, which permanently changes the structure. It is found that this damage threshold can vary by over an order of magnitude for different structures. Illumination powers of around $60 \mathrm{~mW}$ showed severe melting of four out of five structures on their hole arrays and the taper separated irreversibly from the nanowire (see Supporting Information section 3). We have also observed a less severe degree of damage when using lower illumination powers. Here, the images of SHG and THG on the nanowire changed gradually over time. Sometimes, a steady state was reached after a certain exposure time, and the intensity pattern along the nanowire remained unaltered afterward. These nanowires looked visibly unaltered in the SEM, and we expect the changes to be in small roughness features that got "annealed". The general trend that we discovered was that illumination powers of up to $20 \mathrm{~mW}$ could be considered "safe" for most nanowires. The results presented throughout this work are limited to those measurements where no signs of damage were observed on the structures during and after measurements.

We verify the nature of the strong visible signal that we observe to ensure that it is indeed caused by harmonic generation. A typical far-field spectrum of the scattered light, shown in Figure 2a, contains two clear peaks at 775 and 517 $\mathrm{nm}$, nicely corresponding to the expected wavelength of SHG and THG, respectively, for a fundamental wavelength of 1550 $\mathrm{nm}$. Moreover, the small spectral width of the peaks is indicative of nonlinear harmonic generation and not luminescence, which, for gold, also results in the emission of visible light but with a much broader spectral signature. ${ }^{31}$

To ensure that the scattered light results from SHG and THG, we measure its intensity as a function of the incident laser power. The results of this power study are plotted in the inset to Figure 2a on a double-log graph. The nonlinear SHG (THG) is supposed to scale with the second (third) power of the incident light. ${ }^{1}$ Indeed, from a fit to the data in Figure 2a, we find that the slopes of yield of the SHG (red circles) and THG (green circles) are $1.9 \pm 0.1$ and $3.0 \pm 0.1$, respectively. Furthermore, we have also varied the wavelength of the incident beam and confirmed that the second- and thirdharmonic wavelengths are always found at $1 / 2$ and $1 / 3$ of the fundamental (Figure 2b).

Altogether, these results confirm that second and third harmonics are generated by SPPs on the Au NW. Since we know the source of the harmonics to be the fundamental SPPs, what remains to be determined is the physical process by which these harmonics, which we detect, are generated. One plausible cause for the observation of harmonics on the nanowire could be that harmonics is merely generated at the apex, where the plasmonic intensity is the strongest. In principle, the newly generated SPPs at $2 \omega$ and $3 \omega$ themselves could then propagate along the nanowire, without any further need for a source. In this case, the harmonic SPP signals in our experiment at $2 \omega(3 \omega)$ would then exhibit a decay length $l_{\mathrm{d}, 2 \omega(3 \omega)}$ approximately equal to the propagation length $l_{\mathrm{p}, 2 \omega(3 \omega)}$ of the 

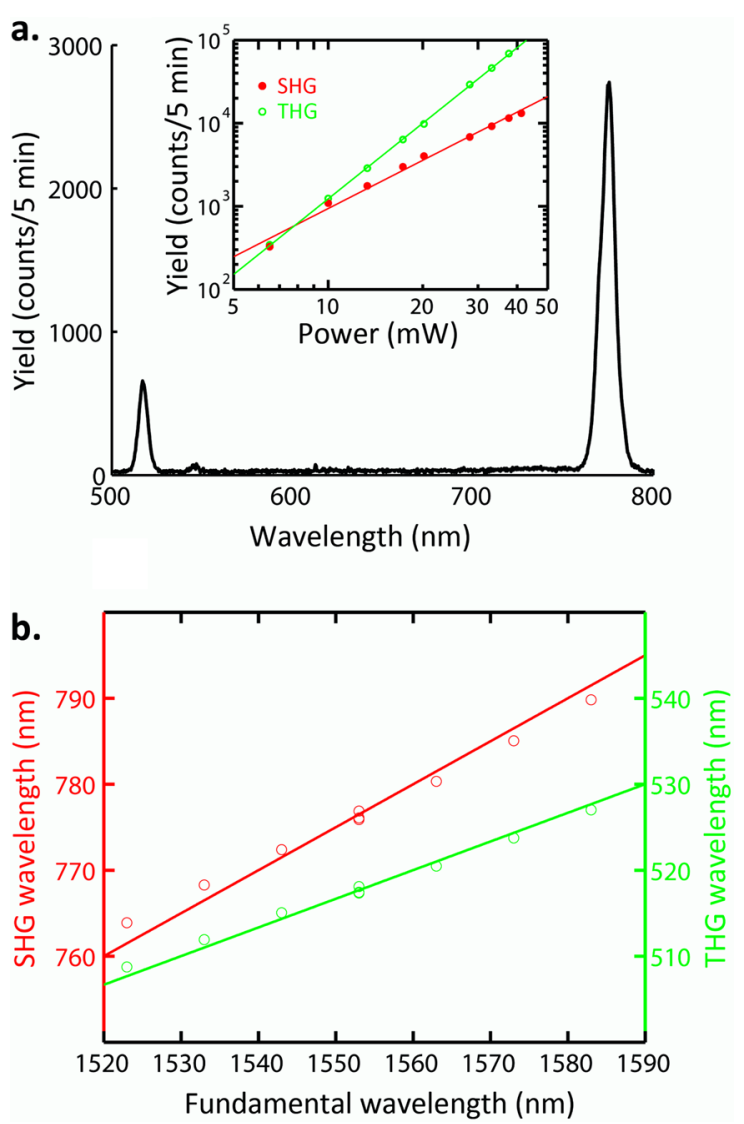

Figure 2. Harmonics generation on a $150 \mathrm{~nm}$ wide Au NW. (a) A typical far-field spectrum exhibits peaks at $775 \mathrm{~nm}$ (SHG) and $517 \mathrm{~nm}$ (THG), for a fundamental wavelength of $1550 \mathrm{~nm}$ and $20 \mathrm{~mW}$ incident power at $3 \mu \mathrm{m}$ from the beginning of the wire. The inset shows the yield (in counts per $5 \mathrm{~min}$ ) for the SHG (red dots) and THG (green open circles) signal as a function of incident laser power. Spectra were collected $1 \mu \mathrm{m}$ from the beginning of the wire. The solid lines are power fits, resulting in slopes of $1.9 \pm 0.1$ and $3.0 \pm 0.1$ for the SHG and the THG data, respectively. (b) A wavelength study shows that the SHG (THG) wavelength shifts as a function of the fundamental incident wavelength in red (green) circles. The red (green) solid lines are linear trends with a slope of $1 / 2(1 / 3)$ of the fundamental wavelength over the range 1520 to $1590 \mathrm{~nm}$.

plasmonic modes at $2 \omega(3 \omega)$ as derived from simulations. From the simulations we know that the propagation lengths for SPPs propagating along a nanowire of $150 \mathrm{~nm}$ wide are $l_{\mathrm{p}, \omega}=11.6 \mu \mathrm{m}, l_{\mathrm{p}, 2 \omega}=2.6 \mu \mathrm{m}$, and $l_{\mathrm{p}, 3 \omega}=37 \mathrm{~nm}$ for the fundamental, second, and third harmonic, respectively. The fact that $l_{\mathrm{p}, 3 \omega} \ll l_{\mathrm{p}, 2 \omega}$ is mainly due to the spectral distance of each respective harmonic to the d-band transition of gold. We find detectable signals for both SHG and THG for all nanowires, many micrometers along the propagation direction, of which an example is presented in Figure 1c for a $150 \mathrm{~nm}$ wide nanowire. In order to collect harmonic signals of the magnitude observed in the experiment, even $\sim 10 \mu \mathrm{m}$ away from the apex, the decay length $l_{\mathrm{d}, 2 \omega(3 \omega)}$ has to greatly exceed $l_{\mathrm{p}, 2 \omega(3 \omega)}$. Hence, the second and third harmonics must be generated along the NW and not merely at its apex.

There are three distinct processes left that can lead to freespace harmonics on our sample, which we sketch in Figure 3a. First, harmonics can be efficiently generated at local field hot spots, caused by imperfections in our sample (process I), resulting in $2 \omega$ photons radiating away from the sample. In this case, we would expect the $\mathrm{SH}$ point spread function to be sharply peaked at the position of the imperfections since this process relies on a local plasmon resonance (LPR).

The other two processes by which harmonics can be generated on the NW, which are denoted processes II and III in Figure $3 \mathrm{a}$, rely on propagating SPP modes and not LPRs. In process II, SHG converts the fundamental SPP to a $2 \omega$ SPP that also propagates on the NW. Due to surface roughness, this $2 \omega$ SPP is scattered into free-space photons that we can detect. Process III, meanwhile, is the direct conversion of fundamental SPPs into $2 \omega$ photons. Because both process II and III rely on propagating waves as sources, we expect that the detected $\mathrm{SH}$ will vary smoothly as a function of position on the NW, with the details of this spatial dependence being determined by the NW geometry. More specifically, we can expect the intensity of the generated second harmonics by process II and III to decay exponentially. The intensity of the fundamental SPPs decays exponentially along the wire, $x$, via $I_{\omega}(x)=I_{0} \mathrm{e}^{-x / l_{\mathrm{p}, \omega}}$, with $l_{\mathrm{p}}(\omega)$ being the propagation length of $\omega$. Because the intensity of the second and third harmonic depends on the intensity of the fundamental quadratically, $I_{2 \omega} \propto I_{\omega}{ }^{2}$, and cubically, $I_{3 \omega} \propto$ $I_{\omega}{ }^{3}$, we expect for processes II and III that the position dependence of the second harmonic is described by $I_{2 \omega}(x)=I_{0} \mathrm{e}^{-x /\left(l_{\mathrm{p}, \omega} / 2\right)}$ and $I_{3 \omega}(x)=I_{0} \mathrm{e}^{-x /\left(l_{\mathrm{p}, \omega} / 3\right)}$. In other words, when guided fundamental plasmons act as the source of second and third harmonics, their decay lengths should be 1/ 2 and $1 / 3$ the propagation length of the fundamental, following $l_{\mathrm{d}, 2 \omega}=\frac{l_{\mathrm{p}, \omega}}{2}$ and $l_{d, 3 \omega}=\frac{l_{p, \omega}}{3}$.

To confirm that the fundamental SPPs act as the source of the harmonics, we derive their decay length $l_{\mathrm{d}, 2 \omega}$ from the decay of the second-harmonic intensity along the nanowire, which should follow $I_{2 \omega}(x) \propto \mathrm{e}^{-x / l_{\mathrm{d}, 2 \omega}}$. Hence, we look for the spatial signature of the harmonic generation processes at low powers where the $\mathrm{SH}$ signal is greater than the $\mathrm{TH}$ signal (see inset to Figure 2a), to avoid damaging the NWs. At these low powers, the $\mathrm{TH}$ signal from the $\mathrm{NW}$ is comparable to that from the glass interface, ${ }^{32}$ and consequently we henceforth restrict ourselves to studies of the $\mathrm{SH}$. An example of the spatial intensity maps of the $\mathrm{SH}$, here for four different $90 \pm 10 \mathrm{~nm}$ wide NWs, is shown in Figure $3 \mathrm{~b}$. Such maps are obtained by integrating the yield maps (e.g., Figure 1c) along the NW width (over 6 pixels along $y, \sim 3 \mu \mathrm{m}$ ), for different $x$ positions along the NW. In this figure, $x=0 \mu \mathrm{m}$ (marked by the dashed line) is the beginning of the NW. Before the NW $(x<0 \mu \mathrm{m})$, as the taper width decreases (sketch above Figure $3 b$ ), we observe that the SHG yield gradually increases to a maximum value near $x=0 \mu \mathrm{m}$. This increase shows that the plasmonic mode on the narrower width sections is better confined, resulting in larger field enhancements (e.g., see Figure 1b) and hence more SHG.

On the NWs we observe two distinct types of features in the $\mathrm{SH}$. At random locations along each $\mathrm{NW}$ we observe strong peaks in the harmonic signal (which are more easily observed on linear plots; see supplementary section 2). As an example, we have marked these peaks with arrows, on the first (red) curve. These, then, are locations where process I occurs, and harmonics are generated at hot spots of defects.

In the SH signal, we also observe large regions where there are no large defects and the harmonic decays smoothly, as expected from processes II and III. For the SH signal of the first 
a.

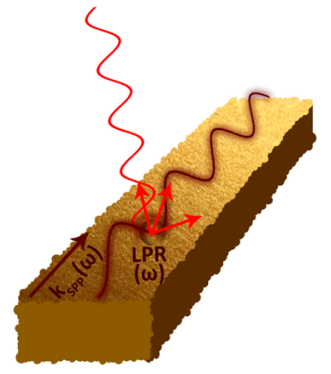

process I

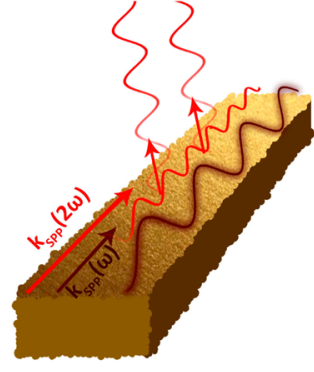

process II

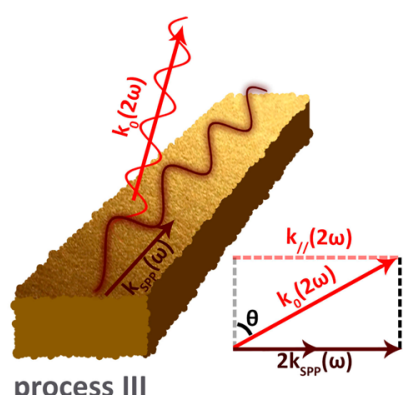

process III

b.
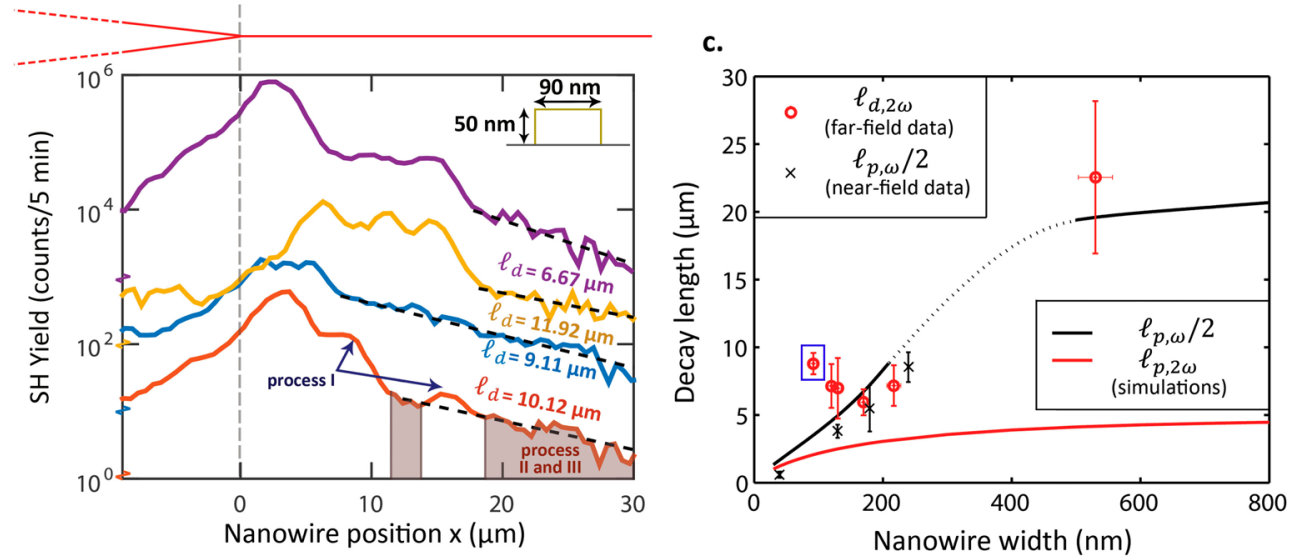

Figure 3. Spatial evolution of the second- harmonic generation on plasmonic nanowires. (a) Three processes that can occur on the NWs, which result in $2 \omega$ photons radiating toward our detector. Process I is the local plasmon resonance (LPR)-enhanced harmonic generation from defects in the sample. Process II is plasmonic second-harmonic generation, while in process III fundamental SPPs are directly converted into $2 \omega$ photons. Process III results in a coherent beam if phase-matching conditions (inset) are fulfilled. (b) Measured SH yield from four nominally identical $90 \mathrm{~nm}$ wide NWs (offset vertically for clarity). The NW begins at $x=0 \mu \mathrm{m}$, as shown in the sketch above the figure and indicated by the dashed gray line. In each curve, we observe peaks, which are indicative of HG via process I, and exponential decay, which is indicative of processes II and III. Fits and the resultant propagation decay, $l_{\mathrm{d}}$, constants for the latter regions are shown for each curve. (c) Extracted $l_{\mathrm{d}}$ of $2 \omega$ as a function of NW width (empty red circles). Also shown are the calculated $l_{\mathrm{p}, 2 \omega}$ and $l_{\mathrm{p}, \omega} / 2$ for $2 \omega$ and $\omega$ SPPs, in red and black curves, respectively. We have included near-field measurements of the decay of the fundamental SPPs (black crosses). Note, however, that we plot $l_{\mathrm{p}, \omega} / 2$ rather than $l_{\mathrm{p}, \omega}$, because of the anticipated $l_{\mathrm{d}, 2 \omega}=\frac{l_{\mathrm{p}, \omega}}{2}$ decay. The dashed region of the black curve corresponds to NW widths where the fundamental SPP mode is leaky.

NW that we show in Figure 3b (red curve), these are marked by shaded regions. In these semilogarithmic plots, the spatial evolution of the SH signal appears linear, indicating that it decays exponentially. We can therefore extract a decay length, $l_{\mathrm{d}, 2 \omega}$, for each NW trace, as is shown in Figure 3b. By averaging over the traces of nominally identical wires, we determine a decay length for each specific wire geometry (see supplementary section 2). In the case of the $90 \mathrm{~nm}$ wide wires shown in Figure $3 \mathrm{~b}$, this decay length is $8.8 \pm 0.8 \mu \mathrm{m}$.

In this manner, we measure $l_{\mathrm{d}, 2 \omega}$ for different width NWs. The results are shown in Figure $3 \mathrm{c}$ as open red circles. In this figure we also give the calculated $l_{\mathrm{p}, 2 \omega}$ for the $2 \omega \mathrm{SPPs}$, as well as $l_{\mathrm{p}, \omega} / 2$ for the $\omega$ SPPs (with red and black curves, respectively). Clearly, the measured $l_{\mathrm{d}, 2 \omega}$ is always much larger than the propagation length of the $2 \omega$ SPPs $l_{\mathrm{p}, 2 \omega}$, yet agrees well with $l_{\mathrm{p}, \omega} / 2$ of the $\omega$ SPPs. We do, however, observe a deviation between our measurements and calculations for the narrowest NWs. We conjecture that this difference could arise from the fact that the quality of the Au could be different for these sub-100 nm wide wires. To validate the behavior of the fundamental SPPs, we included near-field measurements on these same samples of wires (taken from ref 10), which confirms that the calculated $l_{\mathrm{p}, \omega} / 2$ of the fundamental SPPs are correct in the plasmonic region (Figure 3c, black crosses). Indeed, as can be seen from Figure $3 c$, our measurements of $l_{\mathrm{d}, 2 \omega}$ are in agreement with both the near-field measurements and simulations of $l_{\mathrm{p}, \omega} / 2$. This measured spatial dependence of the second-harmonic signal is therefore consistent with a process where the intensity of the generated second harmonic is dependent on the $I(\omega)^{2}$ at that same position. From the data, we therefore conclude that the fundamental SPPs are the source of the observed harmonics, and, indeed, harmonics are generated both from LPRs and SPPs.

To determine which of the two processes, II or III (see Figure 3a), is responsible for the exponentially decaying signal, we consider the wavevectors of the light fields in our system. We show these wavevectors, which are associated with either SPPs or photons (as denoted by the subscripts spp or 0 ), as a function of NW width in Figure 4a. Here, we show the wavevectors for NW widths of up to $200 \mathrm{~nm}$, since for wider NWs these wavevectors are almost constant, as a function of $\mathrm{NW}$ width. In this plot we look for phase matching between the fundamental SPPs, where we consider $2 k_{\text {spp, } \omega}$ (dashed black curve), since SHG requires two fundamental SPPs, and either the second-harmonic SPPs or photons, denoted by $k_{\text {spp }, 2 \omega}$ and $k_{0,2 \omega}$ (red and blue curves, respectively). In other words, phase matching for processes II and III requires geometries where $\Delta k_{i}$ 


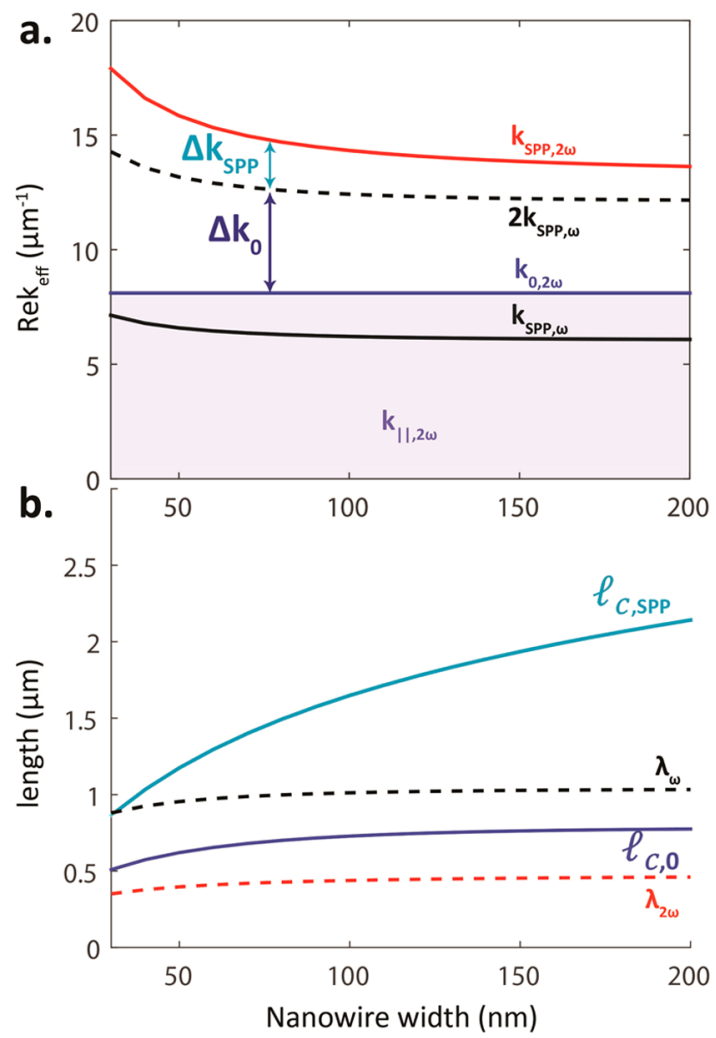

Figure 4. Phase-matching on plasmonic NWs. (a) Real part of the wavevector of the $\omega$ and $2 \omega$ SPPs (black and red curves) and of light in free space (blue curve), as a function of NW width. The respective phase mismatch is marked by $\Delta k$. (b) Coherence lengths of the $\operatorname{SPP}(\omega)$ to $\operatorname{SPP}(2 \omega), l_{\mathrm{c}, \mathrm{spp}}$, and $\operatorname{SPP}(\omega)$ to $\operatorname{photon}(2 \omega), l_{\mathrm{c}, 0}$, as a function of the NW width (solid curves). For comparison, we also show the effective wavelength of the $\omega$ and $2 \omega$ SPPs (dashed curves).

$=2 k_{\text {spp }, \omega}-k_{i, 2 \omega}=0$, where $k_{i, 2 \omega}$ can either be $k_{\text {spp }, 2 \omega}$ or $k_{\|, 2 \omega}=k_{0}$ $\sin \theta$ (as shown in the inset to Figure $3 a$ and a, $2 \omega$ s a purple region in Figure 4a, respectively). In fact, as can be clearly seen in Figure 4a, our NWs do not support phase-matched harmonic generation of either $2 \omega$ photons or plasmons. For completeness, we note that for NW widths between 230 and $670 \mathrm{~nm}$, where the mode is leaky (see Figure 3c), phase matching at near-grazing angles in the glass can occur.

Interestingly, momentum mismatch does not preclude harmonic generation via processes II and III; it simply limits their efficiency. We therefore calculate the coherence length, $l_{c}=\pi / \Delta k$, which is the distance over which the phase of the SH light relative to the fundamental changes by $\pi$ radians. These are shown in Figure $4 \mathrm{~b}$, along with the wavelength of the $2 \omega$ SPPs, $\lambda_{2 \omega}$, and of the $\omega$ SPPs, $\lambda_{\omega}$, for completeness, in dashed curves. Because for these NW widths $\lambda_{2 \omega}$ and $l_{c, 0}$ are comparable and very short, the generated $\mathrm{SH}$ light would undergo a rapid phase change and, in total, there would be no buildup of harmonic amplitude; consequently, we expect that process III (Figure 3a) will be very weak. In contrast, $l_{c, S P P}$ is significantly larger than $\lambda_{2 \omega}$, and hence we expect coherent buildup of $2 \omega$ SPPs. A signature of this coherence would be oscillations in the $\mathrm{SH}$ yield that would be best visible near the beginning of the NW, where the maximum $\mathrm{SH}$ intensity is greatest. Unfortunately, due to the scattering from the random defects, these oscillations were masked in our measurements. We expect that this coherent buildup of $2 \omega$ SPPs would be more visible in near-field measurements. Indeed, preliminary near-field measurements of the spectral content of the light at the surface of the NWs reveal the presence of harmonics. A full near-field study of nonlinear plasmonics on NWs is, unfortunately, beyond the scope of the current work.

\section{CONCLUSION}

In summary, we have shown that guided modes of plasmonic NWs can be used for nonlinear optics. Although our NWs are composed of $\mathrm{Au}$, a centrosymmetric material, we observe clear SH and TH signals. We observe a clear dependence of the spatial evolution of the harmonic signal on the NW geometry. Using these spatial maps and numerical simulations, we explore the possible processes by which the harmonics are generated, showing that scattering from LPR at imperfections and the creation of SH SPPs likely dominate.

In all, the results presented in this article demonstrate the promise of nonlinear optics with guided plasmonic modes, potentially opening a new route toward future applications seeking to exploit nonlinear plasmonics. ${ }^{9}$ In that vein, we suggest that using hybrid plasmonic NWs by adding a layer with large nonlinear coefficients, such as ITO, ${ }^{33}$ could greatly increase the harmonic yield. Likewise, by exploiting the inherently large bandwidths supported by our NWs, ${ }^{10,34}$ ultrashort pulses can be used to increase the peak intensity without increasing the power consumption, again greatly increasing the nonlinear conversion efficiency. Such approaches could perhaps allow for the observation of weaker $\chi^{(2)}$ or $\chi^{(3)}$ nonlinear effects, such as spontaneous parametric downconversion or four-wave mixing, ${ }^{1}$ paving a new route toward the realization of integrated optical circuits for classical or quantum information processing.

\section{ASSOCIATED CONTENT}

\section{S Supporting Information}

The Supporting Information is available free of charge on the ACS Publications website at DOI: 10.1021/acsphotonics.5b00686.

Additional information (PDF)

\section{AUTHOR INFORMATION}

\section{Corresponding Author}

*E-mail (L. Kuipers): kuipers@amolf.nl.

\section{Notes}

The authors declare no competing financial interest.

\section{ACKNOWLEDGMENTS}

The authors thank B. le Feber and R. van der Wel for their insights and help in preparing the manuscript. This work is supported by the NQO program and the research program of the Stichting voor Fundamenteel Onderzoek der Materie (FOM), which is financially supported by the Dutch Organization for Scientific Research (NWO). Part of this work has been funded by the EU FET project "SPANGL4Q". L.K. acknowledges financial support by the ERC Advanced Investigator Grant (no. 240438-CONSTANS).

\section{REFERENCES}

(1) Boyd, R. W. Nonlinear Optics; Academic Press, 2003; Vol. 5.

(2) Byer, R. L. Nonlinear Optics and Solid-State Lasers: 2000. IEEE J. Sel. Top. Quantum Electron. 2000, 6, 911-930. 
(3) Barad, Y.; Eisenberg, H.; Horowitz, M.; Silberberg, Y. Nonlinear Scanning Laser Microscopy by Third Harmonic Generation. Appl. Phys. Lett. 1997, 70, 922-924.

(4) Cotter, D.; Manning, R. J.; Blow, K. J.; Ellis, A. D.; Kelly, A. E.; Nesset, D.; Phillips, I. D.; Poustie, A. J.; Rogers, D. C. Nonlinear Optics for High-Speed Digital Information Processing. Science 1999, 286, 1523-1528.

(5) Corcoran, B.; Monat, C.; Grillet, C.; Moss, D. J.; Eggleton, B. J.; White, T. P.; O'Faolain, L.; Krauss, T. F. Green Light Emission in Silicon through Slow-Light Enhanced Third-Harmonic Generation in Photonic-Crystal Waveguides. Nat. Photonics 2009, 3, 206-210.

(6) Foster, M. A.; Turner, A. C.; Lipson, M.; Gaeta, A. L. Nonlinear Optics in Photonic Nanowires. Opt. Express 2008, 16, 1300-1320.

(7) Monat, C.; de Sterke, M.; Eggleton, B. J. Slow Light Enhanced Nonlinear Optics in Periodic Structures. J. Opt. 2010, 12, 104003.

(8) Chang, D. E.; Vuletić, V.; Lukin, M. D. Quantum Nonlinear Optics - Photon by Photon. Nat. Photonics 2014, 8, 685-694.

(9) Kauranen, M.; Zayats, A. V. Nonlinear Plasmonics. Nat. Photonics 2012, 6, 737-748.

(10) Wulf, M.; Hoogh, A. De; Rotenberg, N.; Kuipers, L. Ultrafast Plasmonics on Gold Nanowires: Confinement, Dispersion, and Pulse Propagation. ACS Photonics 2014, 1, 1173-1180.

(11) Novotny, L.; van Hulst, N. Antennas for Light. Nat. Photonics 2011, 5, 83-90.

(12) Danckwerts, M.; Novotny, L. Optical Frequency Mixing at Coupled Gold Nanoparticles. Phys. Rev. Lett. 2007, 98, 1-4.

(13) Hanke, T.; Cesar, J.; Knittel, V.; Trügler, A.; Hohenester, U.; Leitenstorfer, A.; Bratschitsch, R. Tailoring Spatiotemporal Light Confinement in Single Plasmonic Nanoantennas. Nano Lett. 2012, 12, 992-996.

(14) Valev, V. K.; Baumberg, J. J.; De Clercq, B.; Braz, N.; Zheng, X.; Osley, E. J.; Vandendriessche, S.; Hojeij, M.; Blejean, C.; Mertens, J.; Biris, C. G.; Volskiy, V.; Ameloot, M.; Ekinci, Y.; Vandenbosch, G. A. E.; Warburton, P. A.; Moshchalkov, V. V.; Panoiu, N. C.; Verbiest, T. Nonlinear Superchiral Meta-Surfaces: Tuning Chirality and Disentangling Non-Reciprocity at the Nanoscale. Adv. Mater. 2014, 26, 4074-4081.

(15) Salomon, A.; Zielinski, M.; Kolkowski, R.; Zyss, J.; Prior, Y. Size and Shape Resonances in Second Harmonic Generation from Silver Nanocavities. J. Phys. Chem. C 2013, 117, 22377-22382.

(16) Zhang, Y.; Wen, F.; Zhen, Y.-R.; Nordlander, P.; Halas, N. J. Coherent Fano Resonances in a Plasmonic Nanocluster Enhance Optical Four-Wave Mixing. Proc. Natl. Acad. Sci. U. S. A. 2013, 110, 9215-9219.

(17) Palomba, S.; Danckwerts, M.; Novotny, L. Nonlinear Plasmonics with Gold Nanoparticle Antennas. J. Opt. A: Pure Appl. Opt. 2009, 11, 114030.

(18) van Nieuwstadt, J. A. H.; Sandtke, M.; Harmsen, R. H.; Segerink, F. B.; Prangsma, J. C.; Enoch, S.; Kuipers, L. Strong Modification of the Nonlinear Optical Response of Metallic Subwavelength Hole Arrays. Phys. Rev. Lett. 2006, 97, 1-4.

(19) Simon, H. J.; Mitchell, D. E.; Watson, J. G. Optical SecondHarmonic Generation with Surface Plasmons in Silver Films. Phys. Rev. Lett. 1974, 33, 1531-1534.

(20) Palomba, S.; Novotny, L. Nonlinear Excitation of Surface Plasmon Polaritons by Four-Wave Mixing. Phys. Rev. Lett. 2008, 101, $1-4$.

(21) Neacsu, C. C.; Reider, G. A.; Raschke, M. B. Second-Harmonic Generation from Nanoscopic Metal Tips: Symmetry Selection Rules for Single Asymmetric Nanostructures. Phys. Rev. B: Condens. Matter Mater. Phys. 2005, 71, 1-4.

(22) Schmidt, S.; Piglosiewicz, B.; Sadiq, D.; Shirdel, J.; Lee, J. S.; Vasa, P.; Park, N.; Kim, D. S.; Lienau, C. Adiabatic Nanofocusing on Ultrasmooth Single-Crystalline Gold Tapers Creates a $10-\mathrm{Nm}$-Sized Light Source with Few-Cycle Time Resolution. ACS Nano 2012, 6, 6040-6048.

(23) MacDonald, K. F.; Samson, Z. L.; Stockman, M. I.; Zheludev, N. I. Ultrafast Active Plasmonics. Nat. Photonics 2009, 3, 55-58.
(24) Stockman, M. Nanofocusing of Optical Energy in Tapered Plasmonic Waveguides. Phys. Rev. Lett. 2004, 93, 1-4.

(25) Verhagen, E.; Polman, A.; Kuipers, L. K. Nanofocusing in Laterally Tapered Plasmonic Waveguides. Opt. Express 2008, 16, 4557.

(26) Verhagen, E.; Spasenović, M.; Polman, A.; Kuipers, L. Nanowire Plasmon Excitation by Adiabatic Mode Transformation. Phys. Rev. Lett. 2009, 102, 1-4.

(27) Sipe, J. E.; So, V. C. Y.; Fukui, M.; Stegeman, G. I. Analysis of Second-Harmonic Generation at Metal Surfaces. Phys. Rev. B: Condens. Matter Mater. Phys. 1980, 21, 4389-4402.

(28) Wang, F. X.; Rodríguez, F. J.; Albers, W. M.; Ahorinta, R.; Sipe, J. E.; Kauranen, M. Surface and Bulk Contributions to the SecondOrder Nonlinear Optical Response of a Gold Film. Phys. Rev. B: Condens. Matter Mater. Phys. 2009, 80, 1-4.

(29) Canfield, B.; Kujala, S.; Jefimovs, K.; Turunen, J.; Kauranen, M. Linear and Nonlinear Optical Responses Influenced by Broken Symmetry in an Array of Gold Nanoparticles. Opt. Express 2004, 12, $5418-5423$.

(30) Johnson, P. B.; Christy, R. W. Optical Constants of the Noble Metals. Phys. Rev. B 1972, 6, 4370-4379.

(31) Mooradian, A. Photoluminescence of Metals. Phys. Rev. Lett. $1969,22,185-187$.

(32) Tsang, T. Y. F. Optical Third-Harmonic Generation at Interfaces. Phys. Rev. A: At., Mol., Opt. Phys. 1995, 52, 4116-4125.

(33) Linden, S.; Kuhl, J.; Giessen, H. Controlling the Interaction between Light and Gold Nanoparticles: Selective Suppression of Extinction. Phys. Rev. Lett. 2001, 86, 4688-4691.

(34) Stockman, M. I.; Kling, M. F.; Kleineberg, U.; Krausz, F. Attosecond Nanoplasmonic-Field Microscope. Nat. Photonics 2007, 1, $539-544$. 